\begin{tabular}{lll}
\hline Bentham OPEN & The Open Hypertension Journal \\
CrossMark & Content list available at: www.benthamopen.com/TOHYPERJ/ & DOI: $10.2174 / 1876526201810010046,2018,10,46-51$ \\
\hline
\end{tabular}

LETTER

\title{
Mobile Device for Monitoring of Cardiovascular System State Based on Assessment of Synchronization of its Low-Frequency Rhythms
}

\author{
M.D. Prokhorov ${ }^{1}$, D.D. Kulminskiy ${ }^{1,2}$, E.I. Borovkova ${ }^{2}$, A.S. Karavaev ${ }^{1,2}$, V.I. Ponomarenko ${ }^{1,2}$, A.R. \\ Kiselev $^{2,3, *}$ and B.P. Bezruchko, ${ }^{1,2}$ \\ ${ }^{I}$ Saratov Branch of the Institute of Radio Engineering and Electronics of Russian Academy of Sciences, 38, Zelyonaya \\ str., Saratov410019, Russia \\ ${ }^{2}$ Department of Dynamic Modeling and Biomedical Engineering, Saratov State University; 83, Astrakhanskaya str., \\ Saratov, 410012, Russia \\ ${ }^{3}$ Department of New Cardiological Informational Technologies, Research Institute of Cardiology, Saratov State \\ Medical University n.a. V.I. Razumovsky; 112, Bolshaya Kazachya str, Saratov, 410012, Russia
}

Received: August 5, 2018

Revised: October 11, 2018

Accepted: October 12, 2018

Abstract: We develop an autonomous mobile device for continuous monitoring of cardiovascular system functional state, which is based on the assessment of synchronization between the low-frequency oscillations in heart rate and blood flow having a basic frequency close to $0.1 \mathrm{~Hz}$.

Keywords: Cardiovascular system, Mobile device, Heart rate variability, Photoplethysmogram, Synchronization, Cardiovascular diseases.

\section{INTRODUCTION}

Development of mobile device for interactive medical care in patients with cardiovascular diseases based on contemporary information technologies is an active area of research in recent years [1 - 5]. It has been found out that the human health depends not only on the state of different systems in the organism, but also on the quality of their functional interaction. For example, it was shown that the main rhythmic processes governing the cardiovascular dynamics in humans, namely, the main heart rhythm, respiration, and Low-Frequency (LF) oscillations in heart rate and blood flow generally exhibit epochs of synchronization between themselves, which ensure a high adaptability of the cardiovascular system [6 - 10]. However, this synchronization may be deteriorated at various cardiovascular pathologies, e.g., Acute Myocardial Infarction (AMI) and Hypertension (Htn) leading to a disruption of natural functional couplings within the system of autonomic regulation of cardiovascular system.

In our earlier study [7], we have proposed a method for quantitative estimation of synchronization between the LF oscillations of heart rate and blood flow having in humans a basic frequency close to $0.1 \mathrm{~Hz}$ [11]. It was shown in clinical studies that the proposed method can be used for the choice of drugs for antihypertensive treatment in patients with arterial hypertension [12], for selecting an optimal dose of beta-blocker treatment in patients After Acute Myocardial Infarction (AMI) [13], and for the evaluation of the risk of cardiovascular events in post-AMI patients [14]. Our method was implemented as software intended for the off-line analysis of signals recorded with standard stationary multichannel recorders. The duration of recorded signals in this case is usually rather short.

In the present paper, we develop an autonomous mobile device for continuous recording of photoplethysmogram

\footnotetext{
* Address correspondence to this author at the Department of New Cardiological Informational Technologies, Research Institute of Cardiology, Saratov State Medical University n.a. V.I. Razumovsky; 112, Bolshaya Kazachya str, Saratov, 410012, Russia; Tel: +7 8452 669873; E-mail: kiselev@cardio-it.ru
} 
(PPG) signal and daily monitoring of synchronization between the LF cardiovascular rhythms. We believethat the analysis of data registered over a long period of time can provide a physician with additional useful information about the interaction of oscillating processes governing the cardiovascular dynamics. In prospect, such device can be used for continuous distant control of patient state and help a physician to assign and correct treatment in time.

\subsection{Basic Method of Synchronization Detection}

In our previous studies [7, 12 - 14], synchronization between the LF cardiovascular rhythms has been analyzed using relatively short time series of experimental signals, which duration did not exceed 30 minutes. The signals of Electrocardiogram (ECG) and PPG measured on the middle finger of the subject's hand were simultaneously recorded under spontaneous breathing. The signals were sampled at $250 \mathrm{~Hz}$ and digitized at 14 bits.

The LF oscillations in ECG and PPG signals were extracted in a different way. The LF oscillations in PPG manifest themselves as an amplitude modulation of PPG signal. They were extracted directly from the PPG signal using its bandpass filtration in the range of $0.05-0.15 \mathrm{~Hz}$. The LF oscillations in ECG represent a $0.1-\mathrm{Hz}$ frequency modulation of the main heart rhythm. To extract these oscillations, we first obtained a sequence of RR intervals (RRIs), i.e., a series of time intervals between the two successive R peaks. To obtain equidistant time series from not equidistant sequence of RRIs we approximated it with cubic splines and resample with a frequency of $5 \mathrm{~Hz}$. Then we filtered the sequence of RRIs in the bandpass of $0.05-0.15 \mathrm{~Hz}$. Thus, we excluded the linear mixing of analyzed $0.1-\mathrm{Hz}$ oscillations in ECG and PPG signals. After that we determined the phase $\phi_{1}$ of LF oscillations in PPG and the phase $\phi_{2}$ of LF oscillations in RRIs using the Hilbert transform and calculated the phase difference. To estimate phase synchronization between the considered LF oscillations we used the method based on calculation of synchronization index $S[7,8]$.

\subsection{Method of Synchronization Detection Updated for Mobile Device}

In $[15,16]$ it was shown that synchronization between the different rhythmic processes can be detected even from the analysis of univariate data. Our investigations have shown that it is possible to reject the simultaneous registration of ECG and PPG signals and detect synchronization of LF cardiovascular rhythms from the analysis of only PPG signal. This approach allows us to reduce the device size, weight, and current consumption. Moreover, it considerably simplifies the technical implementation of the device.

In the present paper, we record only PPG signal and obtain the information on Heart Rate Variability (HRV) from the Pulse Rate Variability (PRV). Instead of a sequence of RRIs extracted from ECG, we extract a series of time intervals between the two successive P peaks in PPG and denote it as a sequence of PP Intervals (PPIs). We filter the sequence of PPIs in the bandpass of $0.06-0.14 \mathrm{~Hz}$ to estimate $0.1-\mathrm{Hz}$ oscillations in heart rate. The $0.1-\mathrm{Hz}$ oscillations in PPG are extracted using the same bandpass filtration of PPG signal.

Relationships between HRV and PRV have been investigated in literature [17 - 20]. We found out that the sequence of PPIs closely coincides with the sequence of RRIs (Fig. 1). Since P peaks in PPG are less sharp than R peaks in ECG, their extraction is more difficult. As the result, some PPIs slightly differ from the corresponding RRIs. We compared the values of synchronization index $S$ calculated from bivariate data (ECG and PPG) and univariate data (only PPG) recorded from five healthy subjects and five patients after AMI. The difference between these $S$ values did not exceed $2 \%$ both for healthy subjects and patients. Therefore, the index $S$ can be estimated using only PPG signal.

\subsection{Novel Mobile Device for Monitoring of Cardiovascular System State}

To develop a mobile device for daily monitoring of synchronization of LF oscillations in heart rate and blood flow, one should solve a number of technical problems. The device must have small size, light weight, and low energy consumption. It should provide a quality of signal registration sufficient for detection of synchronization. At first we have developed a prototype, which daily recorded the signals of ECG and PPG. However, it was rather large with the size of 160x80x20 mm, weight greater than $200 \mathrm{~g}$, and current consumption of about $100 \mathrm{~mA}$.

We developed a mobile device with self-contained power supply for 24-hour recording of PPG signal. The device includes a PPG sensor with digital output, elements for control and indication, communication channel for connection to personal computer, flash card, Atmel megaAVR microcontroller controlling the operation of all elements in device, and autonomous power supply system including $3.7 \mathrm{~V} \mathrm{Li-Pol} \mathrm{battery} \mathrm{and} \mathrm{low-dropout} \mathrm{linear} \mathrm{voltage} \mathrm{regulator.} \mathrm{The}$ photo of experimental setup is given in Fig. (2). The device has the size of 90x50x13 mm, weight of $80 \mathrm{~g}$, and current consumption of about $30 \mathrm{~mA}$. 


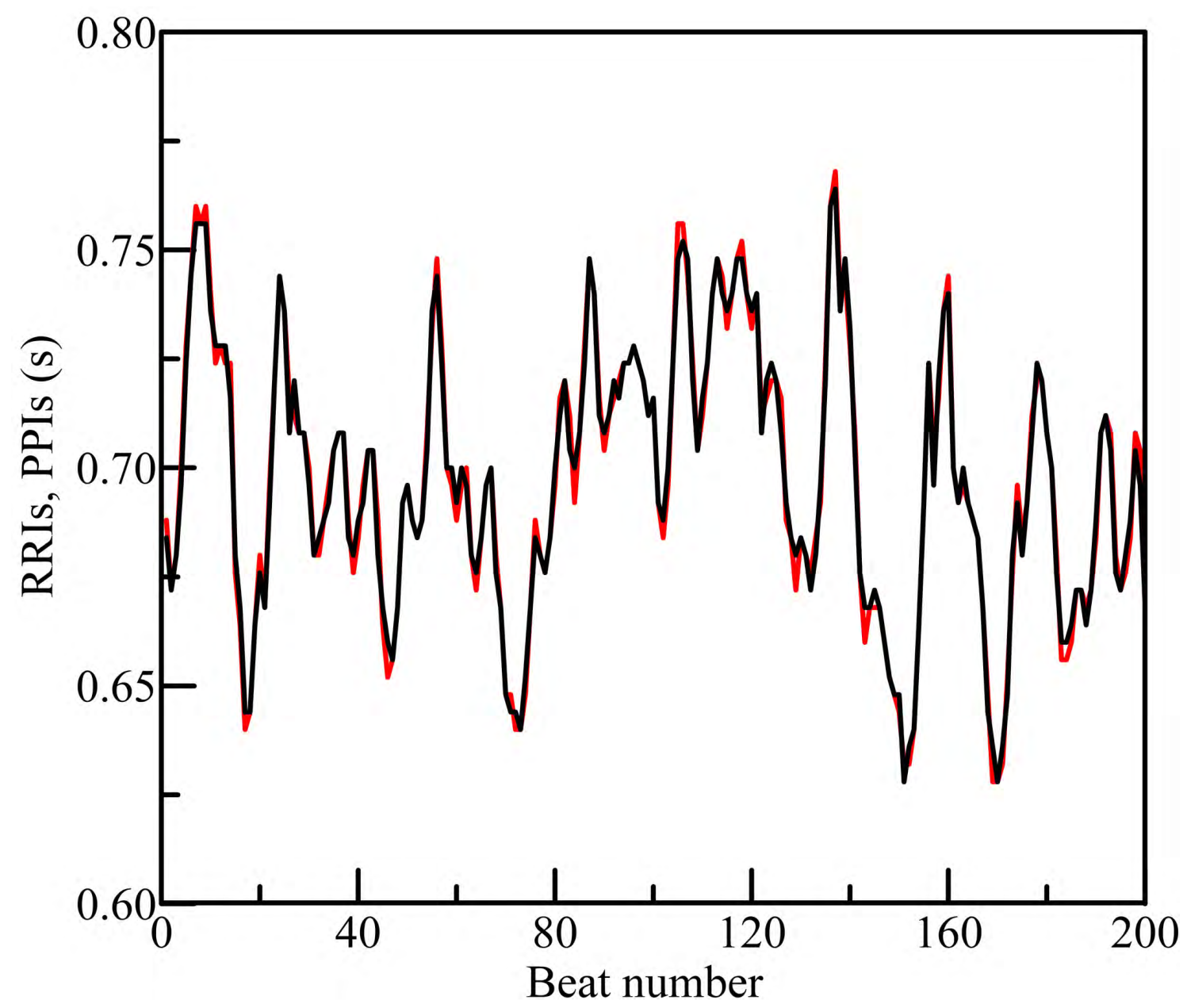

Fig. (1). Comparison of RRIs (black color) and PPIs (red color).

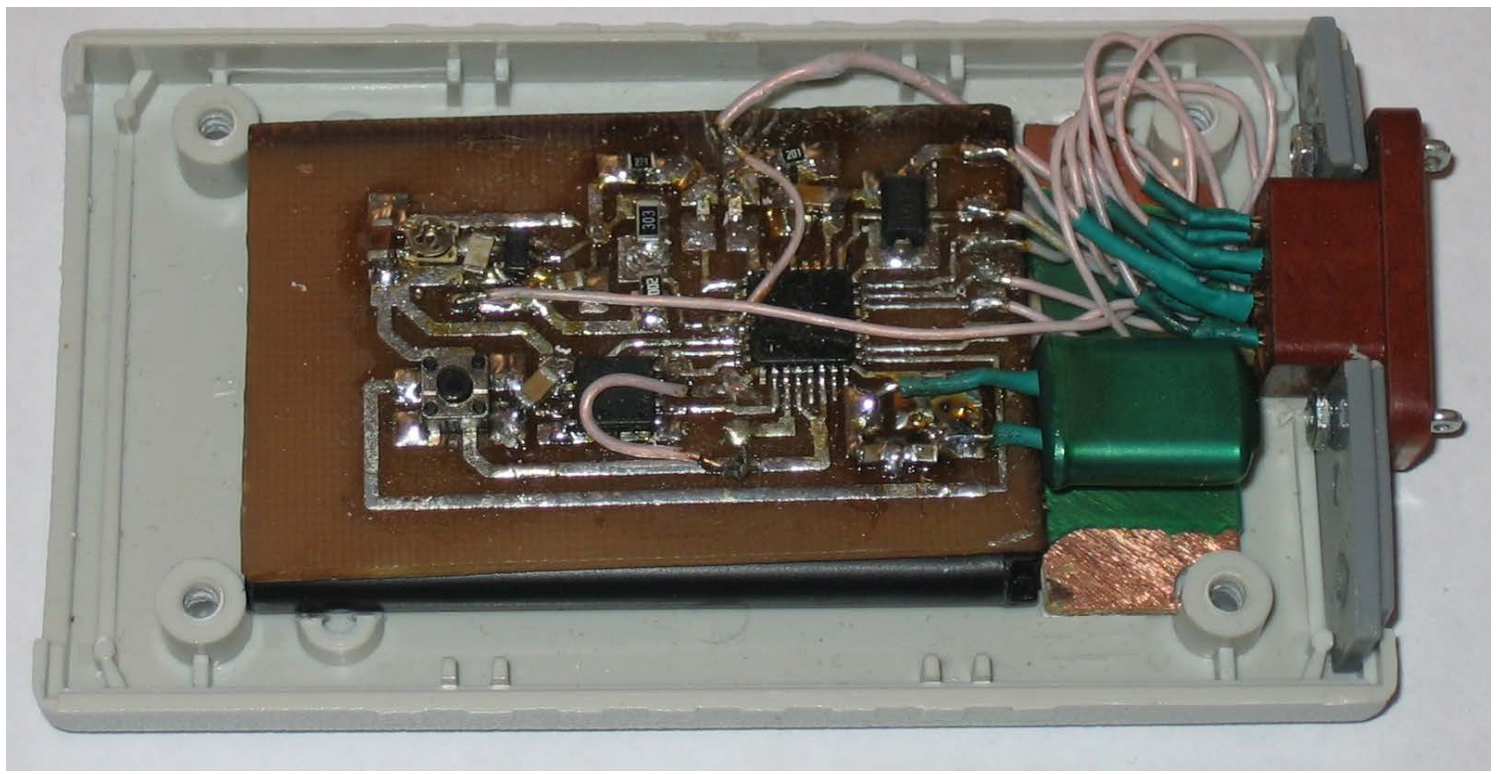

Fig. (2). Photograph of the mobile device. 
The device is placed on the hand and PPG sensor is placed on the finger (Fig. 3). The PPG sensor consists of reflective optocoupler which include an infrared emitter and phototransistor and register finger PPG, 24-bit sigma-delta analog-digital converter, preamplifier, and anti-aliasing filter. The digital output signal of PPG sensor is transmitted to the device via Serial Peripheral Interface. The use of signal quantization directly in the sensor allows us to reduce distortion of the signal during its transmission. We use the whole PPG signal for the analysis.

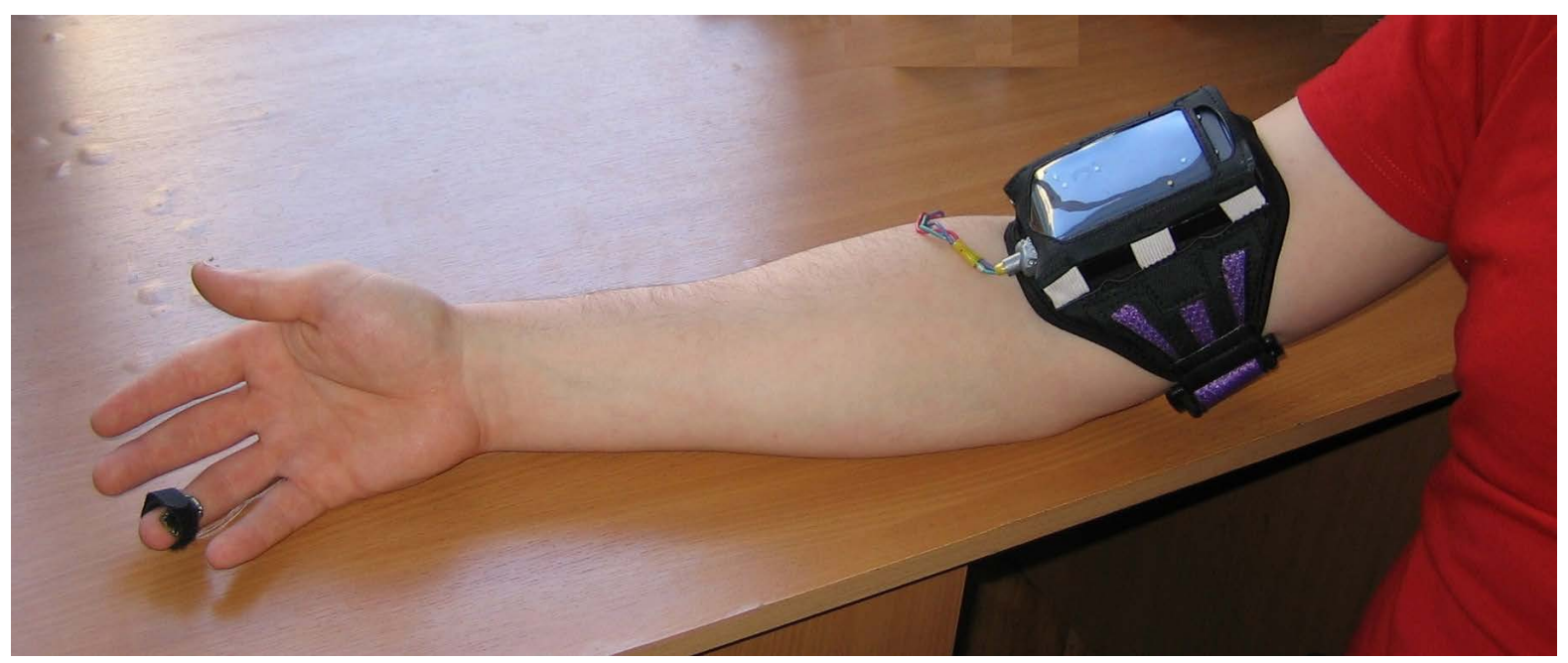

Fig. (3). Placement of device and PPG sensor.

At this stage, the PPG signal is registered and saved onto internal flash card using the developed device and then processed off-line in computer using original software for quantitative assessment of synchronization. The device is connected to personal computer via USB at a speed of $2 \mathrm{Mbit} / \mathrm{s}$.

At the next step we plan to detect synchronization of LF oscillations in RRIs and PPG in real time employing the same microcontroller, which controls the device. To analyze synchronization between these oscillations in real time it is necessary to modify our algorithm of signal processing. In particular, we propose to use a digital finite impulse response (FIR) filter with the bandpass $0.06-0.14 \mathrm{~Hz}$, which can be implemented in real time using microcontroller. To calculate the instantaneous phases of oscillations in real time we plan to implement the Hilbert transform using also discrete digital FIR filter.

We simulated the situation of PPG signal registration in real time and estimated the computational resources required for detecting synchronization of $0.1-\mathrm{Hz}$ cardiovascular rhythms in real time. It has been that technical characteristics of microcontroller used in the developed device allow us to employ this microcontroller for quantitative assessment of synchronization between the LF cardiovascular rhythms in real time.

Further, we plan to carry out both the processes of experimental data recording and processing in the same device. We are going to develop a mobile device for 24-hour recording of PPG signal with simultaneous calculation of degree of synchronization between the $0.1-\mathrm{Hz}$ oscillations in heart rate and blood flow in real time. Much attention will be focused on diminution of size, weight, and energy consumption of the device. It should be noted that finger PPG is sensitive to motion artifacts and accurate detection of peaks in PPG in a moving subject is a challenging problem. In our study, we excluded from the analysis the fragments of PPG signal containing many artifacts. To reduce the influence of motion artifacts, we plan to modify the device in future by adding the option of recording the PPG signal from earlobe.

\section{CONCLUSION}

We have developed the autonomous mobile device for daily recording of PPG signal and original software for calculation of index $S$ characterizing the degree of synchronization between the LF oscillations in heart rate and blood flow. A possibility of detection and quantitative assessment of this synchronization in real time is examined.

\section{ETHICS APPROVAL AND CONSENT TO PARTICIPATE}

Not applicable. 


\section{HUMAN AND ANIMAL RIGHTS}

No animals/humans were used for studies that are the basis of this research.

\section{CONSENT FOR PUBLICATION}

Not applicable.

\section{CONFLICT OF INTEREST}

The authors declare no conflict of interest, financial or otherwise.

\section{ACKNOWLEDGEMENTS}

Declared none.

\section{REFERENCES}

[1] Brucal SG, Clamor GK, Pasiliao LA, Soriano JP, Varilla LP. Portable electrocardiogram device using Android smartphone. Conf Proc IEEE Eng Med Biol Soc 2016; 2016: 509-12.

[2] Dedov VN, Dedova IV. Development of the internet-enabled system for exercise telerehabilitation and cardiovascular training. Telemed J E Health 2015; 21: 575-80. [http://dx.doi.org/10.1089/tmj.2014.0163]

[3] $\mathrm{Hu}$ J, Cui X, Gong Y, et al. Portable microfluidic and smartphone-based devices for monitoring of cardiovascular diseases at the point of care. Biotechnol Adv 2016; 34: 305-20.

[http://dx.doi.org/10.1016/j.biotechadv.2016.02.008]

[4] Sohn K, Merchant FM, Sayadi O, et al. A novel point-of-care smartphone based system for monitoring the cardiac and respiratory systems. Sci Rep 2017; 7: 44946 [http://dx.doi.org/10.1038/srep44946]

[5] Villamil CA, Landínez SF, López DM, Blobel B. A mobile ECG system for the evaluation of cardiovascular risk. Stud Health Technol Inform 2016; $228: 210-4$

[6] Prokhorov MD, Ponomarenko VI, Gridnev VI, Bodrov MB, Bespyatov AB. Synchronization between main rhythmic processes in the human cardiovascular system. Phys Rev E Stat Nonlin Soft Matter Phys 2003; 68: 041913. [http://dx.doi.org/10.1103/PhysRevE.68.041913]

[7] Karavaev AS, Prokhorov MD, Ponomarenko VI, et al. Synchronization of low-frequency oscillations in the human cardiovascular system. Chaos 2009; 19: 033112. [http://dx.doi.org/10.1063/1.3187794]

[8] Kiselev AR, Karavaev AS, Gridnev VI, et al. Method of estimation of synchronization strength between low-frequency oscillations in heart rate variability and photoplethysmographic waveform variability. Russ Open Med J 2016; 5: e0101. [http://dx.doi.org/10.15275/rusomj.2016.0101]

[9] Rosenblum MG, Kurths J, Pikovsky A, Schäfer C, Tass P, Abel HH. Synchronization in noisy systems and cardiorespiratory interaction. IEEE Eng Med Biol Mag 1998; 17: 46-53. [http://dx.doi.org/10.1109/51.731320]

[10] Schäfer C, Rosenblum MG, Kurths J, Abe HH. Heartbeat synchronized with ventilation. Nature 1998; 392: $239-40$. [http://dx.doi.org/10.1038/32567]

[11] Malpas S. Neural influences on cardiovascular variability: Possibilities and pitfalls. Am J Physiol Heart Circ Physiol 2002; 282: H6-H20. [http://dx.doi.org/10.1152/ajpheart.2002.282.1.H6]

[12] Kiselev AR, Gridnev VI, Prokhorov MD, et al. Effects of antihypertensive treatment on cardiovascular autonomic control: A prospective study. Anadolu Kardiyol Derg 2014; 14: 701-10. [http://dx.doi.org/10.5152/akd.2014.5107]

[13] Kiselev AR, Gridnev VI, Prokhorov MD, et al. Selection of optimal dose of beta-blocker treatment in myocardial infarction patients basing on changes in synchronization between $0.1 \mathrm{~Hz}$ oscillations in heart rate and peripheral microcirculation. J Cardiovasc Med (Hagerstown) 2012; 13: $491-8$. [http://dx.doi.org/10.2459/JCM.0b013e3283512199]

[14] Kiselev AR, Gridnev VI, Prokhorov MD, et al. Evaluation of 5-year risk of cardiovascular events in patients after acute myocardial infarction using synchronization of 0.1-Hz rhythms in cardiovascular system. Ann Noninvasive Electrocardiol 2012; 17(3): $204-13$. [http://dx.doi.org/10.1111/j.1542-474X.2012.00514.x]

[15] Janson NB, Balanov AG, Anishchenko VS, McClintock PV. Phase relationships between two or more interacting processes from onedimensional time series. I. Basic theory. Phys Rev E Stat Nonlin Soft Matter Phys 2002; 65: 036211.

[http://dx.doi.org/10.1103/PhysRevE.65.036211] 
[16] Ponomarenko VI, Prokhorov MD, Bespyatov AB, Bodrov MB, Gridnev VI. Deriving main rhythms of the human cardiovascular system from the heartbeat time series and detecting their synchronization. Chaos Solitons Fractals 2005; 23: 1429-38. [http://dx.doi.org/10.1016/S0960-0779(04)00399-6]

[17] Hart J. Association between heart rate variability and manual pulse rate. J Can Chiropr Assoc 2013; 57: 243-50.

[18] Iozzia L, Cerina L, Mainardi L. Relationships between heart-rate variability and pulse-rate variability obtained from video-PPG signal using ZCA. Physiol Meas 2016; 37: 1934-44.

[http://dx.doi.org/10.1088/0967-3334/37/11/1934]

[19] Gil E, Orini M, Bailón R, Vergara JM, Mainardi L, Laguna P. Photoplethysmography pulse rate variability as a surrogate measurement of heart rate variability during non-stationary conditions. Physiol Meas 2010; 31: 1271-90. [http://dx.doi.org/10.1088/0967-3334/31/9/015]

[20] Schäfer A, Vagedes J. How accurate is pulse rate variability as an estimate of heart rate variability? A review on studies comparing photoplethysmographic technology with an electrocardiogram. Int J Cardiol 2013; 166: 15-29. [http://dx.doi.org/10.1016/j.ijcard.2012.03.119]

\section{(c) 2018 Prokhorov et al.}

This is an open access article distributed under the terms of the Creative Commons Attribution 4.0 International Public License (CC-BY 4.0), a copy of which is available at: (https://creativecommons.org/licenses/by/4.0/legalcode). This license permits unrestricted use, distribution, and reproduction in any medium, provided the original author and source are credited. 